\title{
A New Essential Norm Estimate of Composition Operators from Weighted Bloch Space into $\mu$-Bloch Spaces
}

\author{
René E. Castillo, ${ }^{1}$ Julio C. Ramos-Fernández, ${ }^{2}$ and Edixon M. Rojas ${ }^{3}$ \\ ${ }^{1}$ Departamento de Matemáticas, Universidad Nacional de Colombia, AP 360354, Bogotá, Colombia \\ ${ }^{2}$ Departamento de Matemáticas, Universidad de Oriente, Cumaná 6101, Estado de Sucre, Venezuela \\ ${ }^{3}$ Departamento de Matemáticas, Pontificia Universidad Javeriana, Bogotá, Colombia \\ Correspondence should be addressed to Julio C. Ramos-Fernández; jcramos@udo.edu.ve
}

Received 15 May 2013; Accepted 27 August 2013

Academic Editor: Alfonso Montes-Rodriguez

Copyright (c) 2013 René E. Castillo et al. This is an open access article distributed under the Creative Commons Attribution License, which permits unrestricted use, distribution, and reproduction in any medium, provided the original work is properly cited.

Let $\mu$ be any weight function defined on the unit disk $\mathbb{D}$ and let $\phi$ be an analytic self-map of $\mathbb{D}$. In the present paper, we show that the essential norm of composition operator $C_{\phi}$ mapping from the weighted Bloch space to $\mu$-Bloch space $\mathscr{B}^{\mu}$ is comparable to $\lim \sup _{|a| \rightarrow 1^{-}}\left\|\sigma_{a} \circ \phi\right\|_{\mathscr{B}^{\mu}}$, where for $a \in \mathbb{D}, \sigma_{a}$ is a certain special function in the weighted Bloch space. As a consequence of our estimate, we extend the results about the compactness of composition operators due to Tjani (2003).

\section{Introduction}

The weighted Bloch space appears in the literature when we study properties of certain operators acting on certain spaces of analytic functions on the unit disk $\mathbb{D}$ of the complex plane $\mathbb{C}$. For instance, in 1991, Brown and Shields [1] showed that an analytic function $u$ is a multiplier on the Bloch space $\mathscr{B}$ if and only if

$$
\|u\|_{*}=\sup _{z \in D}\left(1-|z|^{2}\right) \log \left(\frac{1}{1-|z|^{2}}\right)\left|u^{\prime}(z)\right|<\infty
$$

Also, in 1992, Attele [2] showed that Hankel operator induced by a function $f \in H(\mathbb{D})$ in the Bergman space $L_{a}^{1}$ (for the definition of Bergman space and the Hankel operator, see [3]) is bounded if and only if $\|f\|_{*}<\infty$, where $H(\mathbb{D})$ is the space of all holomorphic functions on $\mathbb{D}$ with the topology of uniform convergence on compact subsets of $\mathbb{D}$. The set of all functions $f \in H(\mathbb{D})$ such that $(1)$ holds is denoted by $\mathscr{B}^{\log }$ and it is known that $\mathscr{B}^{\log }$ is a Banach space with the norm $\|f\|_{\mathscr{R}^{\log }}=$ $|f(0)|+\|f\|_{*}$.

In the last decade, many authors have studied different classes of Bloch-type spaces, where the weight function, $v(z)=\left(1-|z|^{2}\right) \log \left(1 /\left(1-|z|^{2}\right)\right),(z \in \mathbb{D})$, is replaced by a bounded, continuous, and positive function $\mu$ defined on $\mathbb{D}$.
More precisely, a function $f \in H(\mathbb{D})$ is called a $\mu$-Bloch function, denoted as $f \in \mathscr{B}^{\mu}$, if

$$
\|f\|_{\mu}:=\sup _{z \in \mathbb{D}} \mu(z)\left|f^{\prime}(z)\right|<\infty .
$$

If $\mu_{1}(z)=1-|z|^{2}$, we get the classical Bloch space $\mathscr{B}$ (see [3]), while if $\mu_{2}(z)=\mu_{1}^{\alpha}(z)$ with $\alpha>0, \mathscr{B}^{\mu}$ is just the $\alpha$-Bloch space (see [4]). It is readily seen that $B^{\mu}$ is a Banach space with the norm $\|f\|_{\mathscr{B}^{\mu}}:=|f(0)|+\|f\|_{\mu^{\prime}}$.

A holomorphic function $\phi$ from the unit disk $\mathbb{D}$ into itself induces a linear operator $C_{\phi}$, defined by $C_{\phi}(f)=f \circ \phi$, where $f \in H(\mathbb{D}) . C_{\phi}$ is the so-called composition operator with symbol $\phi$. Composition operators continue to be widely studied on many subspaces of $H(\mathbb{D})$ and particularly in Bloch-type spaces.

The study of the properties of composition operators on Bloch-type spaces began with the celebrated work of Madigan and Matheson in [5], where they characterized the continuity and compactness of composition operators acting on the Bloch space $\mathscr{B}$. Many extensions of Madigan and Matheson's results have appeared (see, e.g., [6] and a lot of references therein). An important reference on this subject is the work of Montes-Rodríguez in [7] (see also [8]); here the author supposes that weight $\mu$ is radial $(\mu(|z|)=\mu(z)$ for all $z \in \mathbb{D})$, typical $\left(\lim _{|z| \rightarrow 1^{-}} \mu(z)=0\right)$, and decreasing function of $|z|$ 
defined on $\mathbb{D}$. In particular, Yoneda in [9] has extended the results by Madigan and Matheson in [5] to composition operators $C_{\phi}$ acting between weighted Bloch spaces. The weight considered by Yoneda in [9] was $\mu_{\log }(z)=\left(1-|z|^{2}\right) \log (2 /(1-$ $\left.|z|^{2}\right)$ ) which is not decreasing on $\mathbb{D}$.

Recently, many authors have found new criteria for the continuity and compactness of composition operators acting on Bloch-type spaces in terms of the $n$th power of the symbol $\phi$ and the norm of the $n$th power of the identity function on $\mathbb{D}$. The first result of this kind appears in 2009 and it is due to Wulan et al. [10]; in turn, their result was extended to $\alpha$-Bloch spaces by Zhao in [11]. Another criterion for the continuity and compactness of composition operators on Bloch space is due to Tjani in [12] (see also [13] or more recently [10]); she showed the following result.

Theorem 1 (see [12]). The composition operator $C_{\phi}$ is compact on $\mathscr{B}$ if and only if $\phi \in \mathscr{B}$ and

$$
\lim _{|a| \rightarrow 1^{-}}\left\|\varphi_{a} \circ \phi\right\|_{\mathscr{B}}=0
$$

where $\varphi_{a}$ is a Möbius transformation from the unit disk onto itself; that is, $\varphi_{a}(z)=(a-z) /(1-\bar{a} z)$, with $z \in \mathbb{D}$.

This last result has been recently extended to $\alpha$-Bloch spaces by Malavé-Ramírez and Ramos-Fernández in [14].

The essential norm of a continuous linear operator $T$ between normed linear spaces $X$ and $Y$ is its distance from the compact operators; that is, $\|T\|_{e}^{X \rightarrow Y}=\inf \left\{\|T-K\|^{X \rightarrow Y}: K\right.$ : $X \rightarrow Y$ is compact $\}$, where $\|\cdot\|^{X \rightarrow Y}$ denotes the operator norm. Notice that $\|T\|_{e}^{X \rightarrow Y}=0$ if and only if $T$ is compact, so that estimates on $\|T\|_{e}^{X \rightarrow Y}$ lead to conditions for $T$ to be compact. The essential norm of a composition operator on $\mathscr{B}$ was calculated by Montes-Rodríguez in [15]. He obtained similar results for essential norms of weighted composition operators between weighted Banach spaces of analytic functions in [7]. Recently, many extensions of the above results have appeared in the literature; for instance, Zhao in [11] gave a formula for the essential norm of $C_{\phi}: \mathscr{B}^{\alpha} \rightarrow \mathscr{B}^{\beta}$ in terms of an expression involving norms of powers of $\phi$. More precisely, he showed that

$$
\left\|C_{\phi}\right\|_{e}^{\mathscr{B}^{\alpha} \rightarrow \mathscr{B}^{\beta}}=\left(\frac{e}{2 \alpha}\right)^{\alpha} \limsup _{j \rightarrow \infty} j^{\alpha-1}\left\|\phi^{j}\right\|_{\mathscr{B}^{\beta}} .
$$

It follows from the discussion at the beginning of this paragraph that $C_{\phi}: \mathscr{B}^{\alpha} \rightarrow \mathscr{B}^{\beta}$ is compact if and only if

$$
\lim _{j \rightarrow \infty} j^{\alpha-1}\left\|\phi^{j}\right\|_{\mathscr{B}^{\beta}}=0 \text {. }
$$

Zhao's results in [11] have been extended recently to the weighted Bloch spaces by Castillo et al. in [16]. Also, Hyvärinen et al. in [17] obtained necessary and sufficient conditions for boundedness and an expression characterizing the essential norm of a weighted composition operator between general weighted Bloch spaces $\mathscr{B}^{\mu}$, under the technical requirements that $\mu$ is radial, and that it is nonincreasing and tends to zero toward the boundary of $\mathbb{D}$.
The goal of the present paper is to give a new estimate of the essential norm of composition $C_{\phi}$ mapping from $\mathscr{B}^{\log }$ to $\mathscr{B}^{\mu}$. More precisely, in the next section we will show the following result.

Theorem 2. Let $\phi$ be an analytic self-map of the unit disk $\mathbb{D}$. Then for the essential norm of the composition operator $C_{\phi}$ : $\mathscr{B}^{\log } \rightarrow \mathscr{B}^{\mu}$, one has

$$
\left\|C_{\phi}\right\|_{e}^{\mathscr{B}^{\log } \rightarrow \mathscr{B}^{\mu}}: \limsup _{|a| \rightarrow 1^{-}}\left\|\sigma_{a} \circ \phi\right\|_{\mathscr{B}^{\mu}} .
$$

The relation (6) means that there is a positive constant $M$ such that

$$
\frac{1}{M}\left\|C_{\phi}\right\|_{e}^{\mathscr{B}^{\log } \rightarrow \mathscr{B}^{\mu}} \leq \limsup _{|a| \rightarrow 1^{-}}\left\|\sigma_{a} \circ \phi\right\|_{\mathscr{B}^{\mu}} \leq M\left\|C_{\phi}\right\|_{e}^{\mathscr{B}^{\log } \rightarrow \mathscr{B}^{\mu}}
$$

and the functions $\sigma_{a}$ with $a \in \mathbb{D}$ will be defined at the beginning of the next section. As a consequence of our estimate, we extend recent results, about the compactness of composition operators, due to Tjani in [12] (see Theorem 1) and MalavéRamírez and Ramos-Fernández in [14].

\section{A Class of Special Functions for $\mathscr{B}^{\log }$}

For convenience, throughout this paper we consider the following weight:

$$
v_{\log }(z)=\left(1-|z|^{2}\right) \log \left(\frac{e}{1-|z|^{2}}\right), \quad(z \in \mathbb{D}),
$$

in place of the one used by Brown and Shields in (1). It is not hard to see that with this new weight we have the same space $\mathscr{B}^{\log }$, and the seminorm

$$
\|f\|_{\log }:=\sup _{z \in \mathbb{D}} v_{\log }(z)\left|f^{\prime}(z)\right|
$$

is comparable to $\|f\|_{*}$ defined in (1). Moreover, this weight $v_{\log }$ satisfies the following property which will be of main relevance in the proof of our result.

Lemma 3. For all $r \in(0,1)$ and all $z \in \mathbb{D}$, one has

$$
r v_{\log }(z) \leq v_{\log }(r z) .
$$

Proof. The result follows from the fact that the function $h(t)=$ $t \log (e / t)$ with $t \in(0,1)$ is concave and satisfies $h(t) \rightarrow 0$ as $t \rightarrow 0^{+}$.

The key to our results lies in considering the following family of functions: for $a \in \mathbb{D}$ fixed, we define

$$
\sigma_{a}(z)=(1-|a|)(H(\bar{a} z)-1), \quad(z \in \mathbb{D}),
$$

where for $a \in \mathbb{D}$, the function $H$ is defined by

$$
H(z)=\frac{1}{(1-z) \log (e /(1-z))} .
$$

We have the following result. 
Lemma 4. The family $\left\{\sigma_{a}\right\}_{a \in \mathbb{D}}$ satisfies the following properties.

(1) $\sigma_{a}$ converges to zero uniformly on compact subsets of $\mathbb{D}$ as $|a| \rightarrow 1^{-}$.

(2) There exists a constant $C_{\sigma}>0$ such that

$$
v_{\log }(a)\left|\sigma_{a}^{\prime}(a)\right| \geq C_{\sigma}
$$

for all $a \in \mathbb{D}$ such that $(1 / 2)<|a|<1$.

(3) There exists a constant $M_{\sigma}>0$ such that

$$
\sup _{a \in \mathbb{D}}\left\|\sigma_{a}\right\|_{\mathscr{R}} \log \leq M_{\sigma} .
$$

Proof. The property (1) is clear since the function $H$ is bounded on compact subsets of $\mathbb{D}$.

(2) If $(1 / 2)<|a|<1$, then

$$
\begin{aligned}
\left|\sigma_{a}^{\prime}(a)\right|= & |a| \frac{(1-|a|) \log \left(1-|a|^{2}\right)}{\left(1-|a|^{2}\right)^{2} \log ^{2}\left(e /\left(1-|a|^{2}\right)\right)} \\
\geq & \frac{1}{2} \frac{\log \left(1-|a|^{2}\right)}{1-\log \left(1-|a|^{2}\right)} \\
& \times \frac{1}{\left(1-|a|^{2}\right) \log \left(1-|a|^{2}\right)},
\end{aligned}
$$

and the property follows since

$$
\lim _{t \rightarrow 0^{+}} \frac{\log (t)}{1-\log (t)}=1 .
$$

(3) Indeed, for $a \in \mathbb{D}$, we have

$$
\begin{aligned}
& \left\|\sigma_{a}\right\|_{\mathscr{B}^{\log }} \\
& =\sup _{z \in \mathbb{D}}|a| \frac{\left(1-|z|^{2}\right) \log \left(e /\left(1-|z|^{2}\right)\right)(1-|a|)|\log (1-\bar{a} z)|}{|(1-\bar{a} z) \log (e /(1-\bar{a} z))|^{2}} \\
& \leq \sup _{z \in \mathbb{D}} \frac{\left(1-|z|^{2}\right) \log \left(e /\left(1-|z|^{2}\right)\right)(1-|a|)|\log (1-\bar{a} z)|}{|1-\bar{a} z|^{2} \log ^{2}(e /|1-\bar{a} z|)}
\end{aligned}
$$

since, for each $w \in \mathbb{C}$, we have $|\log (w)| \geq \log |w|$. Note that the last expression is uniformly bounded if $1 \leq|1-\bar{a} z|<2$, and thus it will be enough to consider the case when $|1-\bar{a} z|<$ 1. Furthermore, since the function $h(t)=t \log (e / t)$ with $t \in$ $(0,1)$ is increasing, concave and satisfy $h(t) \rightarrow 0$ as $t \rightarrow 0^{+}$, then we can use the fact that $1>|1-\bar{a} z| \geq(1 / 2)\left(1-|z|^{2}\right)$ and conclude that

$$
\begin{aligned}
h(|1-\bar{a} z|) & \geq h\left(\frac{1}{2}\left(1-|z|^{2}\right)\right) \\
& \geq \frac{1}{2} h\left(1-|z|^{2}\right) .
\end{aligned}
$$

Hence

$$
\frac{\left(1-|z|^{2}\right) \log \left(e /\left(1-|z|^{2}\right)\right)}{|1-\bar{a} z| \log (e /|1-\bar{a} z|)} \leq 2 .
$$

Also, it is clear that

$$
\frac{1-|a|}{|1-\bar{a} z|} \leq 1
$$

Finally, since

$$
\lim _{t \rightarrow 0^{+}} \frac{\sqrt{\log ^{2} t+4 \pi^{2}}}{1-\log t}=1,
$$

then we can deduce that there exists a constant $M>0$ such

$$
\frac{|\log (1-\bar{a} z)|}{\log (e /|1-\bar{a} z|)} \leq \frac{\sqrt{\log ^{2}|1-\bar{a} z|+4 \pi^{2}}}{1-\log |1-\bar{a} z|} \leq M .
$$

Now the proof is complete.

\section{A Criterion for the Compactness of Operators Acting on Spaces of Analytic Functions}

We will need to prove three auxiliary facts. The first of which is the following lemma that appears in [18] and also in [12]. In both of these places, we point out a typographical error ("point evaluation functionals on $X$ " there, as one can see from Relation (16) in [12], should be "point evaluations on $\left.Y^{\prime \prime}\right)$.

Lemma 5 (Tjani). Let $X, Y$ be two Banach spaces of analytic functions on $\mathbb{D}$. Suppose that

(1) point evaluation functionals on $Y$ are bounded;

(2) the closed unit ball of $X$ is a compact subset of $X$ in the topology of uniform convergence on compact sets;

(3) $T: X \rightarrow Y$ is continuous when $X$ and $Y$ are equipped with the topology of uniform convergence on compact sets.

Then $T$ is a compact operator if and only if given a bounded sequence $\left(f_{j}\right)_{j \in \mathbb{N}}$ in $X$ such that $f_{j} \rightarrow 0$ uniformly on compact sets, $\left(T f_{j}\right)_{j \in \mathbb{N}} \rightarrow 0$ in $Y$.

The following facts about $\mu$-Bloch space are well known.

(i) For each compact $K \subset \mathbb{D}$, there is a $C_{K} \geq 0$ such that for all $f \in \mathscr{B}^{\mu}$ and $z \in K$, we have that

$$
|f(z)| \leq C_{K}\|f\|_{\mathscr{B}^{\mu}} \text {. }
$$

(ii) Every point evaluation functional on $\mathscr{B}^{\mu}$ is bounded.

(iii) The closed unit ball of $\mathscr{B}^{\mu}$ is compact in the topology of uniform convergence on compact subsets of $\mathbb{D}$.

(iv) If $\gamma$ is a weight on $\mathbb{D}$ and $\phi: \mathbb{D} \rightarrow \mathbb{D}$ is holomorphic, with $C_{\phi}: \mathscr{B}^{\mu} \rightarrow \mathscr{B}^{\gamma}$, then $C_{\phi}$ is continuous with respect to the compact-open subspace topologies on $\mathscr{B}^{\mu}$ and $\mathscr{B}^{\gamma}$.

Thus, combining the above and Lemma 5, we obtain the following auxiliary result. 
Lemma 6. Let $\mu_{1}$ and $\mu_{2}$ be weights on $\mathbb{D}$ and suppose that $\phi: \mathbb{D} \rightarrow \mathbb{D}$ is holomorphic. Then $C_{\phi}: \mathscr{B}^{\mu_{1}} \rightarrow \mathscr{B}^{\mu_{2}}$ is compact if and only if given a bounded sequence $\left(f_{j}\right)_{j \in N}$ in $\mathscr{B}^{\mu_{1}}$ such that $f_{j} \rightarrow 0$ uniformly on compact subsets of $\mathbb{D}$, then $\left\|C_{\phi}\left(f_{j}\right)\right\|_{\mathscr{B}^{\mu_{2}}} \rightarrow 0$ as $j \rightarrow \infty$.

As a consequence of Lemma 5, we can see that the dilatation operator is compact on $\mathscr{B}^{\log }$. Recall that for $r \in[0,1]$, the linear dilation operator $K_{r}: H(\mathbb{D}) \rightarrow H(\mathbb{D})$ is defined by $K_{r} f=f_{r}$, where $f_{r}$, for each $f \in H(\mathbb{D})$, is given by $f_{r}(z)=$ $f(r z)$. It is clear that if $f \in H(\mathbb{D})$ then $r f_{r} \rightarrow f$ uniformly on compact subsets of $\mathbb{D}$ as $r \rightarrow 1^{-}$.

Lemma 7. The following statements hold:

(1) for $r \in[0,1)$, the operator $K_{r}$ is compact on $\mathscr{B}^{\log }$;

(2) for each $r \in[0,1]$

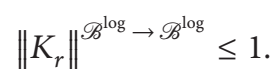

Proof. If $\left\{f_{n}\right\}$ is a bounded sequence in $\mathscr{B}^{\log }$ such that $f_{n} \rightarrow$ 0 uniformly on compact subsets of $\mathbb{D}$, then

$$
\begin{aligned}
0 & \leq \lim _{n \rightarrow \infty}\left\|K_{r} f_{n}\right\|_{\log } \\
& =\sup _{z \in \mathbb{D}} v_{\log }(z) r\left|f^{\prime}(r z)\right| \\
& \leq\left\|v_{\log }\right\|_{\infty} \lim _{n \rightarrow \infty} \sup _{s \in \bar{D}(0, r)}\left|f^{\prime}(s)\right|=0,
\end{aligned}
$$

and the item (1) follows by Lemma 5 .

On the other hand, if $f \in \mathscr{B}^{\log }$ then

$$
\begin{aligned}
\left\|K_{r} f\right\|_{\mathscr{B}^{\log }} & =|f(0)|+\sup _{z \in \mathbb{D}} \frac{r v_{\log }(z)}{v_{\log }(r z)} v_{\log }(r z)\left|f^{\prime}(r z)\right| \\
& \leq|f(0)|+\|f\|_{\log }=\|f\|_{\mathscr{B}^{\log }},
\end{aligned}
$$

where we have used Lemma 3. This ends the proof of item (2).

\section{Proof of Theorem 2}

Now we can show Theorem 2 . We set

$$
L=\limsup _{|a| \rightarrow 1^{-}}\left\|\sigma_{a} \circ \phi\right\|_{\mathscr{B}^{\mu}} \text {. }
$$

Let $K: \mathscr{B}^{\log } \rightarrow \mathscr{B}^{\mu}$ be any compact operator, $a \in \mathbb{D}$ fixed and define

$$
f_{a}(z)=\frac{1}{M_{\sigma}} \sigma_{a}(z), \quad(z \in \mathbb{D}),
$$

where $M_{\sigma}$ is the constant in the item (3) of Lemma 4. Then $f_{a}$ goes to zero uniformly on compact subsets of $\mathbb{D}$ as $|a| \rightarrow 1^{-}$, $\left\|f_{a}\right\|_{\mathscr{B}^{\log }} \leq 1$ for all $a \in \mathbb{D}$, and

$$
\begin{aligned}
\left\|C_{\phi}-K\right\|^{\mathscr{B}^{\log } \rightarrow \mathscr{B}^{\mu}} & \geq\left\|\left(C_{\phi}-K\right) f_{a}\right\|_{\mathscr{B}^{\mu}} \\
& \geq \frac{1}{M_{\sigma}}\left\|\sigma_{a} \circ \phi\right\|_{\mathscr{B}^{\mu}}-\left\|K f_{a}\right\|_{\mathscr{B}^{\mu}} .
\end{aligned}
$$

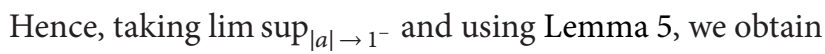

$$
\left\|C_{\phi}\right\|_{e}^{\mathscr{B}^{\log } \rightarrow \mathscr{B}^{\mu}} \geq \frac{1}{M_{\sigma}} \limsup _{|a| \rightarrow 1^{-}}\left\|\sigma_{a} \circ \phi\right\|_{\mathscr{B}^{\mu}} \cdot
$$

Now, we go to show that there exists a constant $C>0$ such that

$$
\left\|C_{\phi}\right\|_{e}^{\mathscr{B}^{\log } \rightarrow \mathscr{B}^{\mu}} \leq C \limsup _{|a| \rightarrow 1^{-}}\left\|\sigma_{a} \circ \phi\right\|_{\mathscr{B}^{\mu}} .
$$

Hence, if we consider a sequence $\left\{r_{n}\right\} \subset(0,1)$ such that $r_{n} \rightarrow 1$ as $n \rightarrow \infty$ and we consider the dilatation operators $K_{n}=K_{r_{n}}$, then by Lemma 7, for all $n \in \mathbb{N}$, the operator $C_{\phi} K_{n}$ is a compact from $\mathscr{B}^{\text {log }}$ into $\mathscr{B}^{\mu}$, and by definition of the essential norm, we have

$$
\left\|C_{\phi}\right\|_{e}^{\mathscr{B}^{\log } \rightarrow \mathscr{B}^{\mu}} \leq \limsup _{n \rightarrow \infty}\left\|C_{\phi}-C_{\phi} K_{n}\right\|^{\mathscr{B}^{\log } \rightarrow \mathscr{B}^{\mu}} .
$$

Thus, we have to show that there exists a constant $M>0$ such that

$$
\limsup _{n \rightarrow \infty}\left\|C_{\phi}-C_{\phi} K_{n}\right\|^{\mathscr{B}^{\log } \rightarrow \mathscr{B}^{\mu}} \leq M L
$$

To see this, consider any $f \in \mathscr{B}^{\log }$ such that $\|f\|_{\mathscr{B}}^{\log }=1$; then since

$$
\begin{aligned}
\left\|\left(C_{\phi}-C_{\phi} K_{n}\right) f\right\|_{\mathscr{B}^{\mu}}= & \left|f(\phi(0))-f\left(r_{n} \phi(0)\right)\right| \\
& +\left\|\left(f-f_{r_{n}}\right) \circ \phi\right\|_{\mu}
\end{aligned}
$$

and $\left|f(\phi(0))-f\left(r_{n} \phi(0)\right)\right| \rightarrow 0$ as $n \rightarrow \infty$, it is enough to show that there exists a constant $M>0$ such that

$$
\limsup _{n \rightarrow \infty}\left\|\left(f-f_{r_{n}}\right) \circ \phi\right\|_{\mu} \leq M L \text {. }
$$

Furthermore, since $r_{n}\left(f^{\prime}\right)_{r_{n}} \rightarrow f^{\prime}$ uniformly on compact subsets of $\mathbb{D}$ as $n \rightarrow \infty$, we have

$$
\limsup _{n \rightarrow \infty} \sup _{|\phi(z)| \leq r_{N}} \mu(z)\left|\left(f-f_{r_{n}}\right)^{\prime}(\phi(z))\right|\left|\phi^{\prime}(z)\right|=0,
$$

where $N \in \mathbb{N}$ is large enough such that $r_{n} \geq(1 / 2)$ for all $n \geq$ $N$. Hence we only have to show that there exists a constant $M>0$ such that

$$
\begin{aligned}
S & :=\limsup _{n \rightarrow \infty} \sup _{|\phi(z)|>r_{N}} \mu(z)\left|\left(f-f_{r_{n}}\right)^{\prime}(\phi(z))\right|\left|\phi^{\prime}(z)\right| \\
& \leq M L .
\end{aligned}
$$

Indeed, we write $S \leq \lim \sup _{n \rightarrow \infty}\left(S_{1}+S_{2}\right)$, where

$$
\begin{aligned}
& S_{1}=\sup _{|\phi(z)|>r_{N}} \mu(z)\left|f^{\prime}(\phi(z))\right|\left|\phi^{\prime}(z)\right|, \\
& S_{2}=\sup _{|\phi(z)|>r_{N}} \mu(z) r_{n}\left|f^{\prime}\left(r_{n} \phi(z)\right)\right|\left|\phi^{\prime}(z)\right| .
\end{aligned}
$$


Then we have

$$
\begin{aligned}
S_{1}= & \sup _{|\phi(z)|>r_{N}} \mu(z)\left|f^{\prime}(\phi(z))\right|\left|\phi^{\prime}(z)\right| \\
& \times \frac{v_{\log }(\phi(z))\left|\sigma_{\phi(z)}^{\prime}(\phi(z))\right|}{v_{\log }(\phi(z))\left|\sigma_{\phi(z)}^{\prime}(\phi(z))\right|} \\
\leq & \frac{1}{C_{\sigma}}\|f\|_{\mathscr{B}}^{\log } \sup _{|\phi(z)|>r_{N}} \mu(z)\left|\sigma_{\phi(z)}^{\prime}(\phi(z))\right|\left|\phi^{\prime}(z)\right| \\
\leq & \frac{1}{C_{\sigma}} \sup _{|\phi(z)|>r_{N}|a|>r_{N}} \sup \mu(z)\left|\sigma_{a}^{\prime}(\phi(z))\right|\left|\phi^{\prime}(z)\right| \\
\leq & \frac{1}{C_{\sigma|a|>r_{N}}} \sup _{\mid \sigma_{a} \circ \phi \|_{\mathscr{B}^{\mu}},}
\end{aligned}
$$

where we have used the item (2) of Lemma 4 in the first inequality and the fact that $\|f\|_{\mathscr{B}^{\log }} \leq 1$ in the second one. Taking limit as $N \rightarrow \infty$, we obtain

$$
\limsup _{n \rightarrow \infty} S_{1} \leq \frac{1}{C_{\sigma}} L .
$$

In a similar way, we have

$$
\begin{aligned}
& S_{2} \leq \frac{1}{C_{\sigma}}\|f\|_{\mathscr{B}^{\log }} \sup _{|\phi(z)|>r_{N}} \mu(z)\left|\sigma_{\phi(z)}^{\prime}(\phi(z))\right| \\
& \quad \times\left|\phi^{\prime}(z)\right| \frac{r_{n} v_{\log }(\phi(z))}{v_{\log }\left(r_{n} \phi(z)\right)} \\
& \leq \frac{1}{C_{\sigma|a|>r_{N}}} \sup _{\left\|\sigma_{a} \circ \phi\right\|_{\mathscr{B}^{\mu}},}
\end{aligned}
$$

where we have used Lemma 3 in the last inequality. Therefore

$$
\left\|C_{\phi}\right\|_{e}^{\mathscr{B}^{\log } \rightarrow \mathscr{B}^{\mu}} \leq \frac{2}{C_{\sigma}} \limsup _{|a| \rightarrow 1^{-}}\left\|\sigma_{a} \circ \phi\right\|_{\mathscr{B}^{\mu}} .
$$

This completes the proof of the theorem.

As an immediate consequence of Theorem 2, we have the following corollary which extends the result due to Tjani in [12].

Corollary 8. The composition operator $C_{\phi}$ is compact from $\mathscr{B}^{\log }$ into $\mathscr{B}^{\mu}$ if and only if $\phi \in \mathscr{B}^{\mu}$ and

$$
\lim _{|a| \rightarrow 1^{-}}\left\|\sigma_{a} \circ \phi\right\|_{\mu}=0
$$

We want to finish this paper with the following question: Is the above corollary true for the composition operator $C_{\phi}$ from $\mathscr{B}^{\mu_{1}}$ into $\mathscr{B}^{\mu_{2}}$, where $\mu_{1}$ and $\mu_{2}$ are weights defined on $\mathbb{D}$ ?

\section{References}

[1] L. Brown and A. L. Shields, "Multipliers and cyclic vectors in the Bloch space," The Michigan Mathematical Journal, vol. 38, no. 1, pp. 141-146, 1991.
[2] K. R. M. Attele, "Toeplitz and Hankel operators on Bergman one space," Hokkaido Mathematical Journal, vol. 21, no. 2, pp. 279293, 1992.

[3] K. H. Zhu, Operator Theory in Function Spaces, vol. 139 of Monographs and Textbooks in Pure and Applied Mathematics, Marcel Dekker, New York, NY, USA, 1990.

[4] K. H. Zhu, "Bloch type spaces of analytic functions," The Rocky Mountain Journal of Mathematics, vol. 23, no. 3, pp. 1143-1177, 1993.

[5] K. Madigan and A. Matheson, "Compact composition operators on the Bloch space," Transactions of the American Mathematical Society, vol. 347, no. 7, pp. 2679-2687, 1995.

[6] J. C. Ramos-Fernández, "Composition operators between $\mu$ Bloch spaces," Extracta Mathematicae, vol. 26, no. 1, pp. 75-88, 2011.

[7] A. Montes-Rodríguez, "Weighted composition operators on weighted Banach spaces of analytic functions," Journal of the London Mathematical Society, vol. 61, no. 3, pp. 872-884, 2000.

[8] M. D. Contreras and A. G. Hernandez-Diaz, "Weighted composition operators in weighted Banach spaces of analytic functions," Journal of the Australian Mathematical Society A, vol. 69, no. 1, pp. 41-60, 2000.

[9] R. Yoneda, "The composition operators on weighted Bloch space," Archiv der Mathematik, vol. 78, no. 4, pp. 310-317, 2002.

[10] H. Wulan, D. Zheng, and K. Zhu, "Compact composition operators on BMOA and the Bloch space," Proceedings of the American Mathematical Society, vol. 137, no. 11, pp. 3861-3868, 2009.

[11] R. Zhao, "Essential norms of composition operators between Bloch type spaces," Proceedings of the American Mathematical Society, vol. 138, no. 7, pp. 2537-2546, 2010.

[12] M. Tjani, "Compact composition operators on Besov spaces," Transactions of the American Mathematical Society, vol. 355, no. 11, pp. 4683-4698, 2003.

[13] H. Wulan, "Compactness of composition operators on BMOA and VMOA," Science in China A, vol. 50, no. 7, pp. 997-1004, 2007.

[14] M. T. Malavé-Ramírez and J. C. Ramos-Fernández, "On a criterion for continuity and compactness of composition operators acting on $\alpha$-Bloch spaces," Comptes Rendus Mathematique, vol. 351, no. 1-2, pp. 23-26, 2013.

[15] A. Montes-Rodríguez, "The essential norm of a composition operator on Bloch spaces," Pacific Journal of Mathematics, vol. 188, no. 2, pp. 339-351, 1999.

[16] R. E. Castillo, D. D. Clahane, J. F. Farías-López, and J. C. RamosFernández, "Composition operators from logarithmic Bloch spaces to weighted Bloch spaces," Applied Mathematics and Computation, vol. 219, no. 12, pp. 6692-6706, 2013.

[17] O. Hyvärinen, M. Kemppainen, M. Lindström, A. Rautio, and E. Saukko, "The essential norm of weighted composition operators on weighted Banach spaces of analytic functions," Integral Equations and Operator Theory, vol. 72, no. 2, pp. 151-157, 2012.

[18] M. Tjani, Compact composition operators on some Möbius invariant Banach space, [Ph. D. dissertation], Michigan State University, East Lansing, Mich, USA, 1996. 


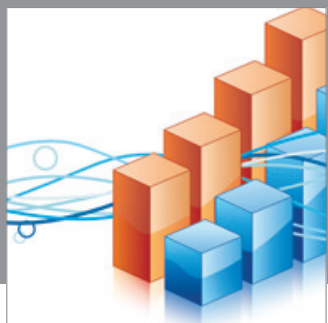

Advances in

Operations Research

mansans

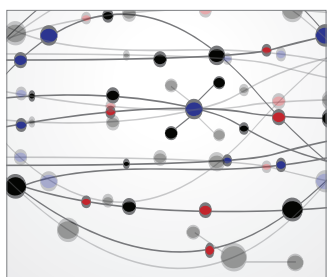

The Scientific World Journal
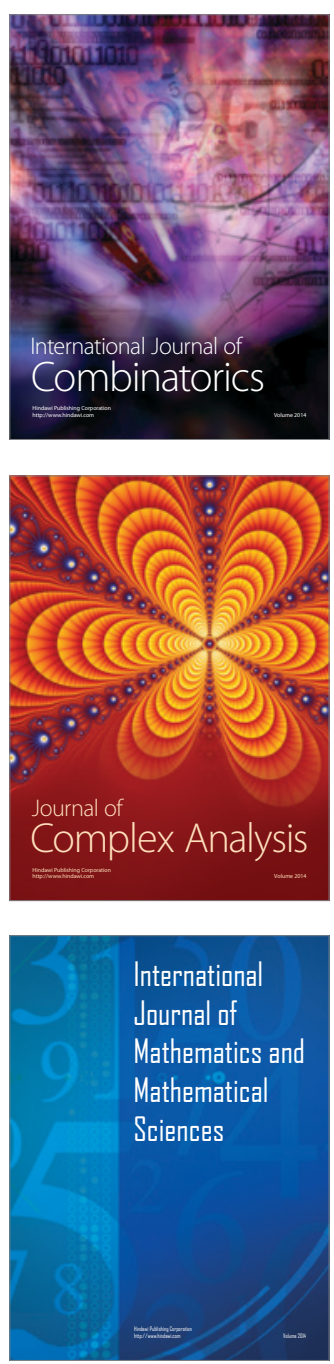
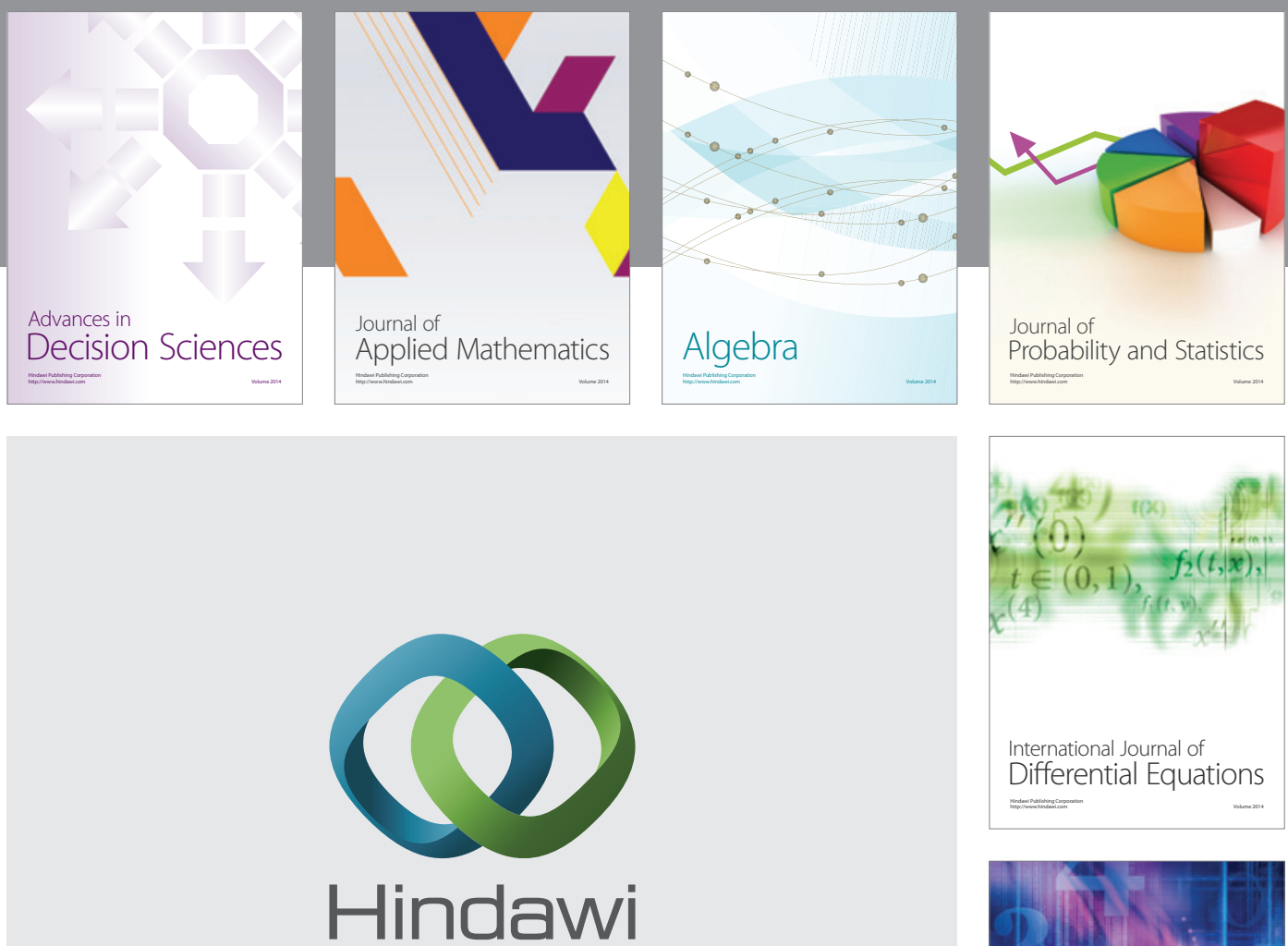

Submit your manuscripts at http://www.hindawi.com
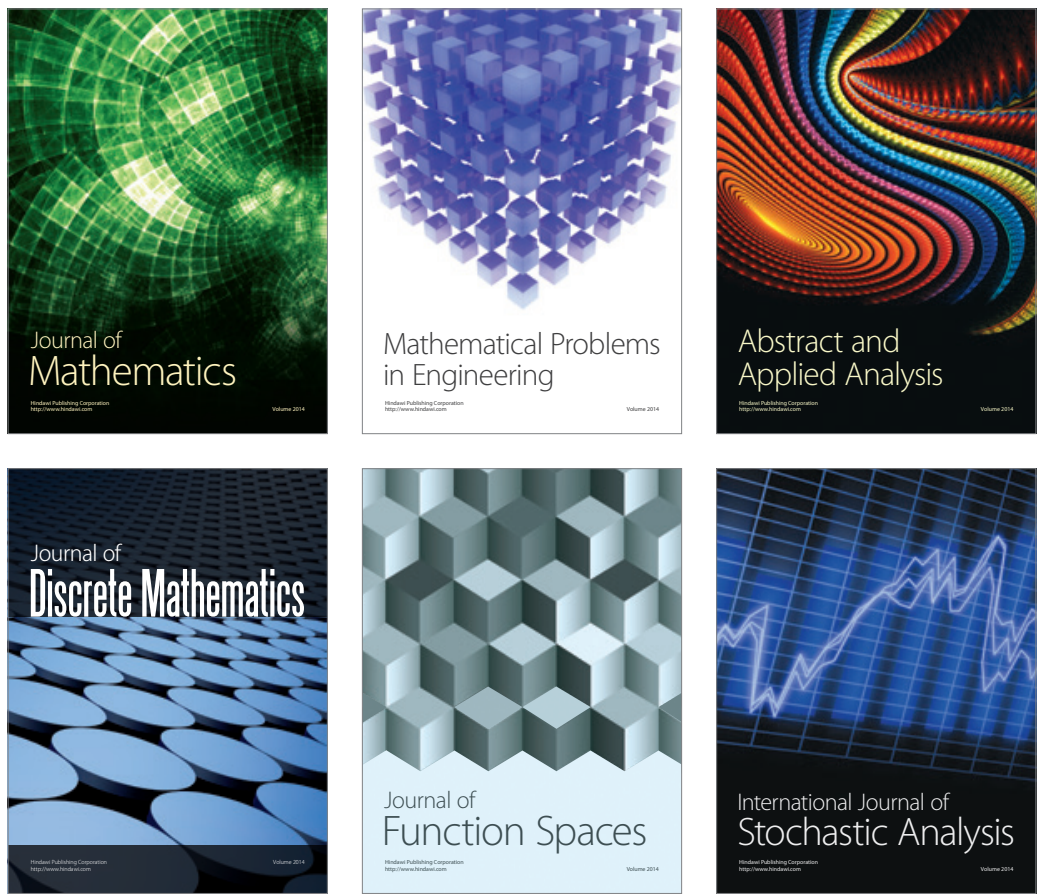

Journal of

Function Spaces

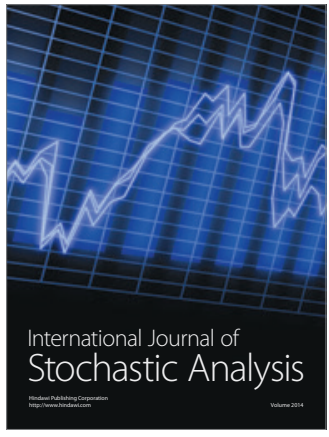

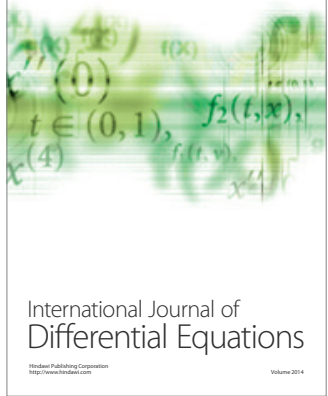
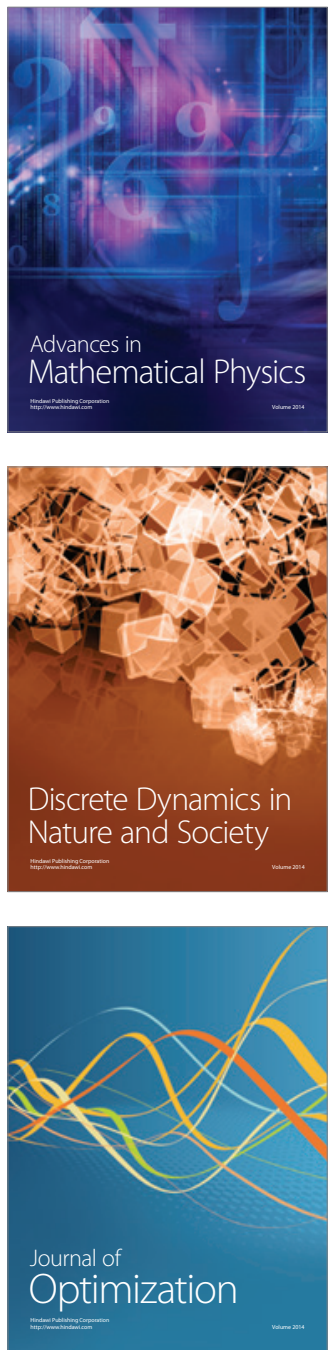